\title{
ON A PROBLEM OF HAYMAN
}

\author{
By XIN-HOU HuA
}

\section{Introduction}

Let $f(z)$ be meromorphic in the complex plane. We will use the following standard notations of Nevanlinna theory,

$$
T(r, f), \quad m(r, f), \quad N(r, f), \quad \bar{N}(r, f), \quad S(r, f), \cdots
$$

(see Hayman [3]).

A meromorphic function $a(z)$ is said to be a small function related to $f$ if

$$
T(r, a)=S(r, f) \text {. }
$$

Hayman [2] proved the following result:

THEOREM A. If $k$ is a positive integer and $f(z)$ is a transcendental meromorphic function in the complex plane, then

$$
T(r, f)<\left(2+\frac{1}{k}\right) N\left(r, \frac{1}{f}\right)+\left(2+\frac{2}{k}\right) \bar{N}\left(r, \frac{1}{f^{(k)}-1}\right)+S(r, f) .
$$

Hayman [3, p. 73] asked whether the coefficients of $\boldsymbol{N}(r, 1 / f)$ and $\bar{N}\left(r, 1 / f^{(k)}-1\right)$ are best possible, where $\bar{N}\left(r, 1 / f^{(k)}-1\right)$ is the counting function of the roots of $f^{(k)}-1=0$ in $|z| \leqq r$, multiple roots been counted once.

Concerning this problem, Frank and Hennekemper [1] proved the following:

THEOREM B. Let $f(z)$ be a meromorphic function which has only simple poles $k \geqq 2, c \in C \backslash\{0\}, f \not \equiv$ constant and $f^{(k)}-c \neq \equiv$. Then

$$
T(r, f) \leqq N\left(r, \frac{1}{f}\right)+\left(1+\frac{2}{k-1}\right) \bar{N}\left(r, \frac{1}{f^{(k)}-c}\right)+S(r, f) .
$$

In this paper, we shall prove tho following result:

THEOREM 1. Suppose that $f(z)$ is transcendental and meromorphic in the complex plane, and that $k$ is a positive integer. If $p(z)$ is a nonzero polynomial or a nonzero constant, then for any $\varepsilon>0$, we have

Received January 16, 1990; revise April 13, 1990. 


$$
T(r, f) \leqq\left(1+\frac{1}{k}+\varepsilon\right)\left\{N\left(r, \frac{1}{f}\right)+N\left(r, \frac{1}{f^{(k)}-p}\right)\right\}+S(r, f) .
$$

Remark. Here $S(r, f)$ depends on $\varepsilon>0$, but the associated exceptional set is independent of $\varepsilon$.

THEOREM 2. Let $f(z)$ be a nonconstant rational function, and let $k$ be a positive integer. If $c \in C \backslash\{0\}$ and $f^{(k)}-c \neq 0$, then we have

$$
T(r, f) \leqq N\left(r, \frac{1}{f}\right)+N\left(r, \frac{1}{f^{(k)}-c}\right)+O(1) .
$$

\section{Some lemmas} theory.

In our first lemma we recall some of the basic relations of Nevanlinna

LEMMA 1 ([3]). Suppose that $f$ and $g$ are nonzero meromorphic functions in the plane. Then for any positive integer $i$, we have

$$
\begin{gathered}
m\left(r, f^{(i)} / f\right)=S(r, f), \\
T\left(r, f^{(i)}\right) \leqq(i+1) T(r, f)+S(r, f) .
\end{gathered}
$$

In addition

$$
N\left(r, \frac{f}{g}\right)-N\left(r, \frac{g}{f}\right)=N(r, f)+N\left(r, \frac{1}{g}\right)-N(r, g)-N\left(r, \frac{1}{f}\right) .
$$

LEMMA 2 (Steinmetz [4, Theorem 1]). Let the linear differential operator

$$
L(y)=y^{(q)}+a_{q-1}(z) y^{(q-1)}+\cdots a_{0}(z) y
$$

have rational coefficients $a_{0}, \cdots, a_{q-1}$ and let $f$ be a transcendental meromorphic function in the plane. Then either $f$ is a rational function of a (local) fundamental set $y_{1}, \cdots, y_{q}$ of the differential equation $L(y)=0$ or inequality

$$
m\left(r, \frac{1}{L(f)}\right) \leqq m(r, L(f))+(1+\eta) N(r, f)+S(r, f)
$$

holds for every $\eta>0$.

LEMMA 3. Suppose that $f(z)$ is meromorphic in $\boldsymbol{C}$, and that $f^{(k)}(z)$ is nonconstant. Then for any small function $a(z)$ related to $f(a \neq 0, \infty)$, we have

$$
\begin{aligned}
T(r, f) \leqq & \bar{N}(r, f)+N\left(r, \frac{1}{f}\right)+N\left(r, \frac{1}{f^{(k)}-a}\right) \\
& -N\left(r, \frac{1}{a f^{(k+1)}-a^{\prime} f^{(k)}}\right)+S(r, f) .
\end{aligned}
$$


Proof. From the identity

$$
\frac{1}{f}=\frac{1}{a}\left\{\frac{f^{(k)}}{f}-\left(a \frac{f^{(k+1)}}{f}-a^{\prime} \frac{f^{(k)}}{f}\right) \frac{f^{(k)}-a}{a f^{(k+1)}-a^{\prime} f^{(k)}}\right\}
$$

and Lemma 1 and $T(r, a)=S(r, f)$ it follows that

$$
\begin{aligned}
m\left(r, \frac{1}{f}\right) \leqq & m\left(r, \frac{f^{(k)}-a}{a f^{(k+1)}-a^{\prime} f^{(k)}}\right)+S(r, f) \\
= & m\left(r, \frac{a f^{(k+1)}-a^{\prime} f^{(k)}}{f^{(k)}-a}\right)+N\left(r, \frac{a f^{(k+1)}-a^{\prime} f^{(k)}}{f^{(k)}-a}\right) \\
& -N\left(r, \frac{f^{(k)}-a}{a f^{(k+1)}-a^{\prime} f^{(k)}}\right)+S(r, f) \\
= & m\left(r, a\left(\frac{f^{(k)}}{a}-1\right)^{\prime} /\left(\frac{f^{(k)}}{a}-1\right)\right)+N\left(r, a f^{(k+1)}-a^{\prime} f^{(k)}\right) \\
& +N\left(r, \frac{1}{f^{(k)}-a}\right)-N\left(r, f^{(k)}-a\right)-N\left(r, \frac{1}{a f^{(k+1)}-a^{\prime} f^{(k)}}\right) \\
& +S(r, f) \\
\leqq & \bar{N}(r, f)+N\left(r, \frac{1}{f^{(k)}-a}\right)-N\left(r, \frac{1}{a f^{(k+1)}-a^{\prime} f^{(k)}}\right) \\
& +S\left(r, f^{(k)}\right)+S(r, f) .
\end{aligned}
$$

Now from (2) we have

$$
S\left(r, f^{(k)}\right)=S(r, f) .
$$

The conclusion follows from (4), (5) and $T(r, f)=m(r, 1 / f)+N(r, 1 / f)+O(1)$.

LEMMA 4. Let $t \geqq 2$ be an arbitrary integer. Suppose that $f(z)$ is transcendental and meromorphic in the complex plane, and that $q(z)$ is a nonzero polynomial. Then for any $\eta>0$ we have

$$
t \bar{N}(r, f) \leqq N\left(r, \frac{1}{q f^{(t)}-q^{\prime} f^{(t-1)}}\right)+(1+\eta) N(r, f)+S(r, f) .
$$

Proof. Let $h(z)$ be a solution of the linear differential equation

$$
L(y)=0 \text {, }
$$

where

$$
L(y)=q y^{(t)}-q^{\prime} y^{(t-1)} .
$$

If $h^{(t-1)} \neq 0$, then from (6) and (7) we deduce that

$$
h^{(t)} / h^{(t-1)}=q^{\prime} / q .
$$


Thus there exists a nonzero constant $c$ such that

which gives

$$
h^{(t-1)}=c q \text {, }
$$

$$
h(z)=q^{*}(z),
$$

where $q^{*}(z)$ is a polynomial of degree $\operatorname{deg}(q)+t-1$.

If $h^{(t-1)} \equiv 0$, then $h(z)$ is a polynomial of degree $t-2$ or less. Let

$$
h_{t}(z)=q^{*}(z), \quad h_{j}(z)=z^{j-1} \quad(j=1, \cdots, t-1) .
$$

Then $\left\{h_{1}(z), \cdots, h_{t}(z)\right\}$ is a (local) fundamental solution set of $L(y)=0$. Since $f(z)$ is transcendental, the solutions $h_{i}(z)(i=1, \cdots, t)$ are small functions related to $f$. Thus, by Lemma $2, L(f) \not \equiv 0$ and

$$
m\left(r, \frac{1}{L(f)}\right) \leqq m(r, L(f))+(2+\eta) N(r, f)+S(r, f)
$$

It follows form (8) and the first fundamental theorem $[3$, p. 5] that

$$
\begin{aligned}
N(r, L(f)) & =T\left(r, \frac{1}{L(f)}\right)-m(r, L(f))+O(1) \\
& \leqq N\left(r, \frac{1}{L(f)}\right)+(2+\eta) N(r, f)+S(r, f) .
\end{aligned}
$$

It is easy to verify that

$$
\begin{aligned}
N(r, L(f)) & =N\left(r, q f^{(t)}-q^{\prime} f^{(t-1)}\right. \\
& \geqq N(r, f)+t \bar{N}(r, f)-O(\log r) .
\end{aligned}
$$

Lemma 3 follows from this and (9).

\section{Proof of Theorem 1}

Applying Lemma 4 to $t=k+1, \eta=\left(\varepsilon k^{2} / k+\varepsilon k+1\right)$ and $q=p$ we have

$$
\begin{aligned}
\bar{N}(r, f) & \leqq \frac{1}{k+1} N\left(r, \frac{1}{p f^{(k+1)}-p^{\prime} f^{(k)}}\right)+\left(1-\frac{k}{k+\varepsilon k+1}\right) N(r, f)+S(r, f) \\
& \leqq \frac{1}{k+1} N\left(r, \frac{1}{p f^{(k+1)}-p^{\prime} f^{(k)}}\right)+\frac{\varepsilon k+1}{k+\varepsilon k+1} T(r, f)+S(r, f) .
\end{aligned}
$$

On the other hand, Lemma 3 gives

$$
\begin{aligned}
T(r, f)< & \bar{N}(r, f)+N\left(r, \frac{1}{f}\right)+N\left(r, \frac{1}{f^{(k)}-p}\right) \\
& -N\left(r, \frac{1}{p f^{(k+1)}-p^{\prime} f^{(k)}}\right)+S(r, f) .
\end{aligned}
$$


Combining this with (10) we derive that

$$
\begin{aligned}
T(r, f) \leqq & \left(1+\frac{1}{k}+\varepsilon\right)\left\{N\left(r, \frac{1}{f}\right)+N\left(r, \frac{1}{f^{(k)}-p}\right)\right\} \\
& -\left(1-\frac{\varepsilon}{k+1}+\varepsilon\right) N\left(r, \frac{1}{p f^{(k+1)}-p^{\prime} f^{(k)}}\right)+S(r, f) .
\end{aligned}
$$

This is what we need.

Remark 1. By a simple calculation and using Example (i) in $[3$, p. 6] we can prove Theorem 2.

Remark 2. Since writing this paper I have learned (through Professor Yuzan $\mathrm{He}$ and correspondence) of progress made by Lo Yang, where Yang proved a result which is similar to Theorem 1 for constant $p$. I wish to thank both for their comments.

Acknowledgement. I am grateful to Professor Chitai Chuang for his advice and I wish to thank the referee for many valuable suggestions.

\section{REFERENCES}

[1] G. FRANK AND W. Hennekemper, Einige Ergebnisse über die Werteverteilung meromorpher Funktionen und ihrer Ableitungen, Resultate Math., 4 (1981), 39-54.

[2] W.K. HAYMAN, Picard values of meromorphic functions and their derivatives, Ann. of Math. 70 (1959), 9-42.

[3] W.K. Hayman, Meromorphic functions (Oxford Univ. Press, 1964).

[4] N. Steinmetz, On the zeros of a certain Wronskian, Bull. London Math. Soc. (5) 20 (1988), 525-531.

Department of Mathematics

Peking University

BeIJING 100871, P.R. China

Current Address

Department of Mathematics

NANJING UNIVERSITY

NANJING 210008

P.R. CHINA 